\title{
BRS Analysis from Baroreflex Sequences and Baroreflex Events Compared Using Spontaneous and Drug Induced Data
}

\author{
S Gouveia ${ }^{1,2}$, AP Rocha ${ }^{1,2}$, P Laguna $^{3,4}$, M Gujic $^{5}$, SP Beloka ${ }^{5}$, P Van de Borne ${ }^{5}$, P Lago $^{1}$ \\ ${ }^{1}$ Departamento de Matemática Aplicada, Faculdade de Ciências, Universidade do Porto, Portugal \\ ${ }^{2}$ Centro de Matemática da Universidade do Porto, Portugal \\ ${ }^{3}$ Comm Tech Group, Aragon Institute of Eng Research, University of Zaragoza, Spain \\ ${ }^{4}$ CIBER de Bioingeniería, Biomateriales y Nanomedicina (CIBER-BBN), Spain \\ ${ }^{5}$ Department of Cardiology, Erasme Hospital, Brussels, Belgium
}

\begin{abstract}
Spontaneous time domain BRS estimation is based on the SBP-RR slope, which can be computed from either baroreflex sequences (BS) or baroreflex events (BE). BRS analysis from BEs was recently shown be advantageous particularly in the cases of reduced BRS or when BS are not identified. Also, it offers a superior discrimination between lying and standing positions.

In this work, the methods developed for spontaneous $B R S$ analysis are further compared using spontaneous and drug induced data. The results corroborate that spontaneous and drug induced estimates are different although correlated. In particular, if BEs are used the differences and the correlation between the estimates is higher. No precision improvement is achieved if the BRS is estimated from drug induced data. In spontaneous, the higher number of beats in BEs in comparison with BSs (at the expense of a lower SBP-RR correlation) allows a higher BRS estimate precision using recordings of the same length.
\end{abstract}

\section{Introduction}

Over the past years, the arterial-cardiac barorereflex sensitivity (BRS) quantification has been useful in the study of many pathological states, including myocardial infarction, hypertension and congestive heart failure [1]. Briefly, lower levels of BRS have been associated with an increased cardiovascular disease-related mortality .

Time domain BRS is traditionally quantified as the regression slope obtained from the cross-analysis between systolic blood pressure (SBP) and RR interval values, either using drug induced or spontaneous data. The advantages and limitations of these techniques have been reviewed elsewhere [2]. Briefly, the noninvasive nature of the spontaneous methods simplifies the test procedure and allows the BRS measurement under a broad range of daily life conditions, making these methods more appropriate in many research settings. The principle of the invasive technique is to make use of a drug which changes arterial pressure by producing vasoconstriction (or vasodilatation) while not having direct effect on the RR interval, so that the observed RR changes are mediated reflexively via the baroreceptors. In comparison with the spontaneous, drug induced techniques stimulate a larger and clearer SBP increase (or decrease) in order to force a pronounced RR response (i.e, a clearer baroreflex activation). Several comparisons between spontaneous and drug induced BRS estimates evidence that they are different although being correlated (e.g, [3]). Therefore spontaneous BRS estimates can have the same clinical and predictive power as the invasive, even beyond that provided by heart rate variability indices alone [4].

The most frequently used spontaneous BRS method is the sequences technique, which is based on the identification of baroreflex sequences (BS) followed by the BRS estimation as the mean of the SBP-RR slopes computed in each BS [5]. The events technique, recently proposed to improve spontaneous BRS assessment, consists of a global slope estimation from the SBP-RR values in baroreflex events (BE) [6]. The BRS analysis from BEs has shown to be advantageous as it provides a larger number of beats for slope estimation, allowing BRS analysis in cases where BSs cannot be identified. However, the higher number of beats is obtained at the expense of a lower SBPRR correlation (although being close to 0.8 ) with possible repercussion on the precision of the BRS estimate.

In this study, a precision measure over time domain BRS estimates from global slope estimation is proposed. Also, the BRS analysis from BSs and from BEs, previously proposed for spontaneous BRS assessment, are further compared using spontaneous and drug induced data. The methods performance is evaluated in terms of the ability to discriminate different conditions and in terms of precision. 


\section{Time domain BRS estimation}

The methods for global BRS estimation combined either with BSs or BEs have been previously detailed [6] and are exemplified in Fig. 1. As illustrated in 1(a) and (b), both BSs and BEs are identified considering $x_{\mathrm{SBP}}(n)$ paired with $x_{\mathrm{RR}}(n+1)$, with $n$ being the beat number. Briefly, each valid $\mathrm{BS}_{k}$ requires a minimum beat length $\left(N_{k} \geq 3\right)$, minimum SBP and RR beat-to-beat changes in the same direction $\left(\Delta_{k}^{\mathrm{SBP}} \geq 1 \mathrm{mmHg}\right.$ and $\left.\Delta_{k}^{\mathrm{RR}} \geq 5 \mathrm{~ms}\right)$ and a minimum $x_{\mathrm{SBP}}$ and $x_{\mathrm{RR}}$ correlation $\left(r_{k} \geq 0.8\right)$, whereas for the identification of each $\mathrm{BE}_{k}$ only the thresholds $N_{\min }$ and $r_{\min }$ are enforced. After the identification, the mean is removed from $x_{\mathrm{SBP}}$ and $x_{\mathrm{RR}}$ at each segment and the result concatenated in $d_{\mathrm{SBP}}$ and $\mathbf{d}_{\mathrm{RR}}$ vectors, respectively. Finally, the global slope $\mathcal{B}_{\mathrm{G}, \mathrm{O}}$ is obtained from the regression analysis $\mathbf{d}_{\mathrm{RR}}=\mathcal{B}_{\mathrm{G}, \mathrm{O}} \mathbf{d}_{\mathrm{SBP}}+\boldsymbol{\epsilon}$, where the parameter $\mathcal{B}_{\mathrm{G}, \mathrm{O}}$ is estimated by ordinary least squares (OLS) minimization and $\epsilon$ a noise vector. Additional variables can be retrieved from global BRS analysis, namely the number of points for slope estimation $(N)$ and the $\mathbf{d}_{\mathrm{SBP}}-\mathbf{d}_{\mathrm{RR}}$ correlation $(r)$. Figure $1(\mathrm{c}-\mathrm{d})$ presents the $\mathbf{d}_{\mathrm{SBP}}$ and $\mathbf{d}_{\mathrm{RR}}$ dispersion diagrams for the example in $(\mathrm{a}-\mathrm{b})$, illustrating that BRS analysis from BEs provides a higher $N$ at the expense of a lower $r$, as a consequence of the less restrictive identification thresholds.

(a)

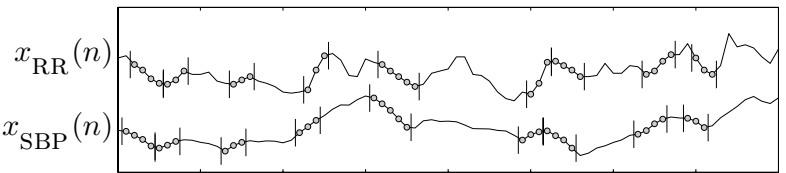

(b)

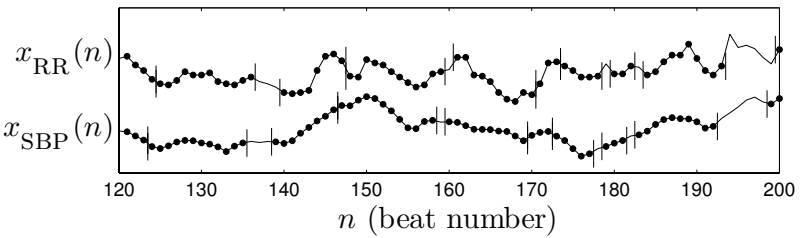

(c)
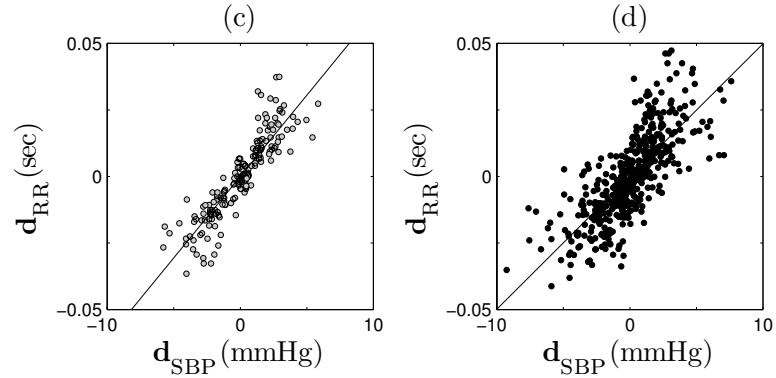

Figure 1. Example of BRS estimation from BSs (a,c) and from BEs $(b, d)$ in a 512 beats spontaneous recording: $(a, b)$ $x_{\mathrm{SBP}}$ and $x_{\mathrm{RR}}$ plot for the identified baroreflex segments and (c,d) corresponding $\mathbf{d}_{\mathrm{SBP}}$ and $\mathbf{d}_{\mathrm{RR}}$ dispersion diagrams with the global regression line with slope $\hat{\mathcal{B}}_{\mathrm{G}, 0}$. For BSs, $N=171$ and $r=0.89$ and for BEs $N=448$ and $r=0.76$.

\subsection{BRS confidence intervals}

Standard parametric confidence intervals (CI) over the regression slope can provide a measure of precision, if the regression residuals $(\hat{\boldsymbol{\epsilon}})$ satisfy some requirements (namely, non correlation, zero mean, homoscedasticity and normal distribution). In this study, the precision was computed from a nonparametric bootstrap CI [7], as the $\hat{\boldsymbol{\epsilon}}$ analysis did not support these assumptions, except for the zero mean normal distribution (with 95\% confidence).

The linear regression was computed from 1000 Bootstrap (B) replications of $\mathbf{d}_{\mathrm{RR}}$ following $\mathbf{d}_{\mathrm{RR}}^{\mathrm{B}}=\hat{\mathcal{B}}_{\mathrm{G}, \mathrm{O}} \mathbf{d}_{\mathrm{SBP}}+\hat{\boldsymbol{\epsilon}}^{\mathrm{B}}$, with $\hat{\mathcal{B}}_{\mathrm{G}, \mathrm{O}}$ the OLS estimate of $\mathcal{B}_{\mathrm{G}, \mathrm{O}}$ and $\hat{\boldsymbol{\epsilon}}^{\mathrm{B}}$ a realization of $\epsilon$ reproducing the mean, variance, autocorrelation and heteroscedastic pattern in $\hat{\boldsymbol{\epsilon}}$. The limits of the $95 \%$ CI were defined by the 2.5th and 97.5 th percentiles of the empirical distribution of the bootstrap slope estimates $\hat{\mathcal{B}}_{\mathrm{G}, \mathrm{O}}^{\mathrm{B}}$. Finally, the $\hat{\mathcal{B}}_{\mathrm{G}, \mathrm{O}}$ relative maximum error $(\delta)$, a measure inversely related to precision, was calculated as the CI half amplitude divided by $\hat{\mathcal{B}}_{\mathrm{G}, \mathrm{O}}$.

For the $\hat{\boldsymbol{\epsilon}}^{\mathrm{B}}$ simulation, it was considered $\hat{\boldsymbol{\epsilon}}=\hat{\boldsymbol{\epsilon}}_{\mathrm{C}}+\hat{\epsilon}_{\mathrm{H}}$, with $\hat{\boldsymbol{\epsilon}}_{\mathrm{C}}$ being the constant variance component and $\hat{\boldsymbol{\epsilon}}_{\mathrm{H}}$ the remaining part (i.e, the heteroscedastic pattern, maintained constant in all realizations). The $\hat{\boldsymbol{\epsilon}}_{\mathrm{C}}$ component was estimated from weighted LS regression [7], considering the weights $w_{i}=\left(\left|p_{i}-1\right| / s_{p}+1\right)^{-1}, i=1,2, \ldots N$ with $p_{i}=\hat{\mathcal{B}}_{i} / \hat{\mathcal{B}}, \hat{\mathcal{B}}_{i}$ the OLS regression slope excluding the $i^{t h}\left(\mathbf{d}_{\mathrm{SBP}}, \mathbf{d}_{\mathrm{RR}}\right)$ pair and $s_{\mathbf{p}}$ the standard deviation of $\mathbf{p}=$ $\left[\begin{array}{llll}p_{1} & p_{2} & \ldots & p_{N}\end{array}\right]$. The bootstrapped residuals $\hat{\boldsymbol{\epsilon}}_{\mathrm{C}}^{\mathrm{B}}$ were generated as white gaussian noise with the same mean and variance as $\hat{\boldsymbol{\epsilon}}_{\mathrm{C}}$. Its autocorrelation was introduced by means of filtering, after $\hat{\boldsymbol{\epsilon}}_{\mathrm{C}}$ autoregressive modeling (minimum AIC order, from Yule-Walker equations and Levinson-Durbin algorithm [8]). The remaining part was obtained by $\hat{\epsilon}_{\mathrm{H}}=\hat{\epsilon}-$ $\hat{\boldsymbol{\epsilon}}_{\mathrm{C}}$ and set as zero for $|\hat{\boldsymbol{\epsilon}}|$ lower than 1.96 times the $\hat{\boldsymbol{\epsilon}}_{\mathrm{C}}$ standard deviation (i.e, non significative heteroscedasticity). Finally, each noise realization was obtained from $\hat{\epsilon}^{\mathrm{B}}=\hat{\epsilon}_{\mathrm{C}}^{\mathrm{B}}+\hat{\epsilon}_{\mathrm{H}}$. Figure 2 illustrates the dispersion diagram of two bootstrap replicas, showing the similarity with the dispersion diagrams in Fig. 1(c-d). (a)

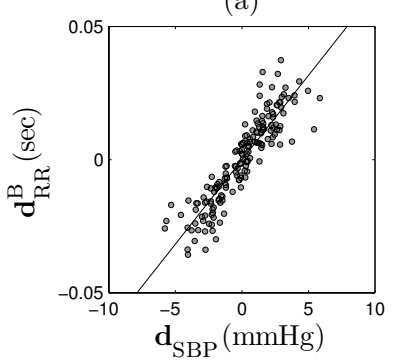

(b)

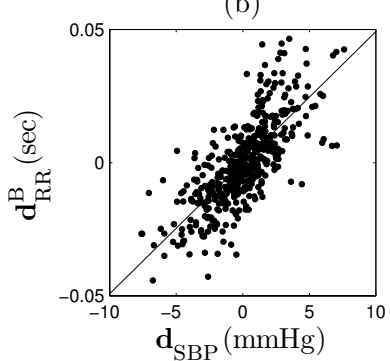

Figure 2. Dispersion diagrams of two bootstrap replicas $(\mathrm{a}-\mathrm{b})$ reproducing the real data in Figure 1(c-d), with the global regression line with slope $\hat{\mathcal{B}}_{\mathrm{G}, \mathrm{O}}^{\mathrm{B}}$. 


\section{Experimental data}

The data was collected from 15 healthy male subjects (20-36 years) in supine rest condition [9]. Each subject was monitored $5 \mathrm{~min}$ in spontaneous (SP) condition and during the 3 min modified Oxford protocol. The latter consists of a bolus injection of $150 \mu \mathrm{g}$ sodium nitroprusside (NT) followed $1 \mathrm{~min}$ later by a bolus of $150 \mu \mathrm{g}$ phenylephrine $\mathrm{HCl}(\mathrm{PH})$. Figure 3 presents the data from one subject, illustrating that the consecutive NT (vasodilator) and $\mathrm{PH}$ (vasoconstrictor) bolus acutely decrease/increase SBP and produce a baroreflex mediated shortening/lengthening of the RR interval, respectively. Also, it can be observed that in this protocol there is a short time gap between the sucessive injections and the $\mathrm{PH}$ is administrated still under the NT effect, leading to a mixture of effects. Therefore, this experimental setting increases the range of SBP changes observed in SP acquisition, but only by lowering the $x_{\mathrm{SBP}}$ and $x_{\mathrm{RR}}$ values with respect to the baseline.

In this work, the BRS in SP was assessed from the 5 min recordings, whereas in NT and $\mathrm{PH}$ it was computed from consecutive $45 \mathrm{sec}$ segments starting $30 \mathrm{sec}$ after the NT bolus (Fig. 2). The $30 \mathrm{sec}$ lag assures that the NT effect is present, as its onset of action is within $30 \mathrm{sec}$ [10], whereas $45 \mathrm{sec}$ is the maximum time interval for all subjects between the beginning of NT and PH effects.

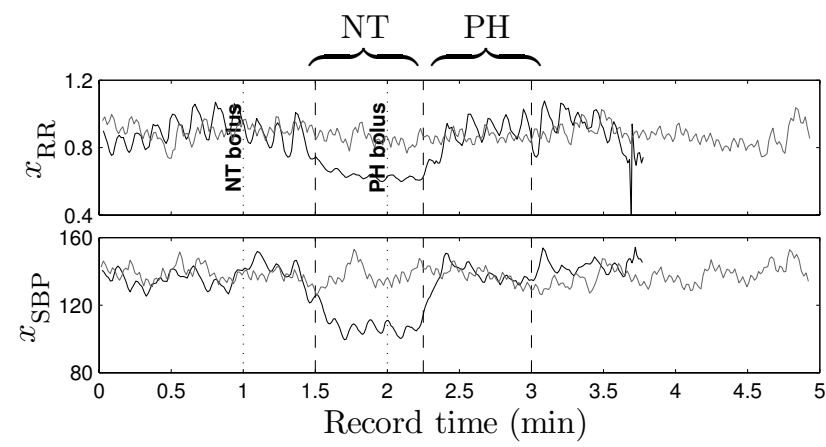

Figure 3. Plot of $x_{\mathrm{SBP}}$ and $x_{\mathrm{RR}}$ in SP (grey) and during the modifed Oxford method (black). Dotted lines identify the timing of NT and PH bolus and the brackets delimitate the time intervals for BRS analysis in NT and PH conditions.

\section{Results}

The methods were compared with respect to $\hat{\mathcal{B}}$ and $\delta$ was studied as a function of $N$ and $r$. Indices $(\mathrm{S}, \mathrm{E})$ and (SP,NT,PH) were added to each variable according to its evaluation: e.g., $\hat{\mathcal{B}}_{\mathrm{SP}}^{\mathrm{E}}$ refers to $\hat{\mathcal{B}}$ from BEs in SP.

It is expected that RR changes after induced SBP increase or decrease are asymmetrical, being the RR response to falling SBP lower than of that to rising SBP [11]. Figure 4 shows the comparison between the $\hat{\mathcal{B}}$. For BEs, $\hat{\mathcal{B}}_{\mathrm{SP}}^{\mathrm{E}}>\hat{\mathcal{B}}_{\mathrm{NT}}^{\mathrm{E}}$ for $15 / 15$ subjects, $\hat{\mathcal{B}}_{\mathrm{SP}}^{\mathrm{E}}>\hat{\mathcal{B}}_{\mathrm{PH}}^{\mathrm{E}}$ for $9 / 15$ and $\hat{\mathcal{B}}_{\mathrm{PH}}^{\mathrm{E}}>\hat{\mathcal{B}}_{\mathrm{NT}}^{\mathrm{E}}$ for $14 / 15$. With BSs, the countings are $11 / 15,4 / 15$, and $13 / 15$, respectively. The mean paired differences $\hat{\mathcal{B}}_{\mathrm{SP}}-\hat{\mathcal{B}}_{\mathrm{NT}}$ and $\hat{\mathcal{B}}_{\mathrm{NT}}-\hat{\mathcal{B}}_{\mathrm{PH}}$ differ from zero $(\mathrm{p}<0.01)$, whereas $\hat{\mathcal{B}}_{\mathrm{SP}}-\hat{\mathcal{B}}_{\mathrm{PH}}$ do not $(\mathrm{p}>0.1)$. The pairwise correlation between $\hat{\mathcal{B}}_{\mathrm{SP}}, \hat{\mathcal{B}}_{\mathrm{NT}}$ and $\hat{\mathcal{B}}_{\mathrm{PH}}$ is higher for BEs $(r>0.85$ and $\mathrm{p}<0.01$ for the null hypothesis of no correlation).
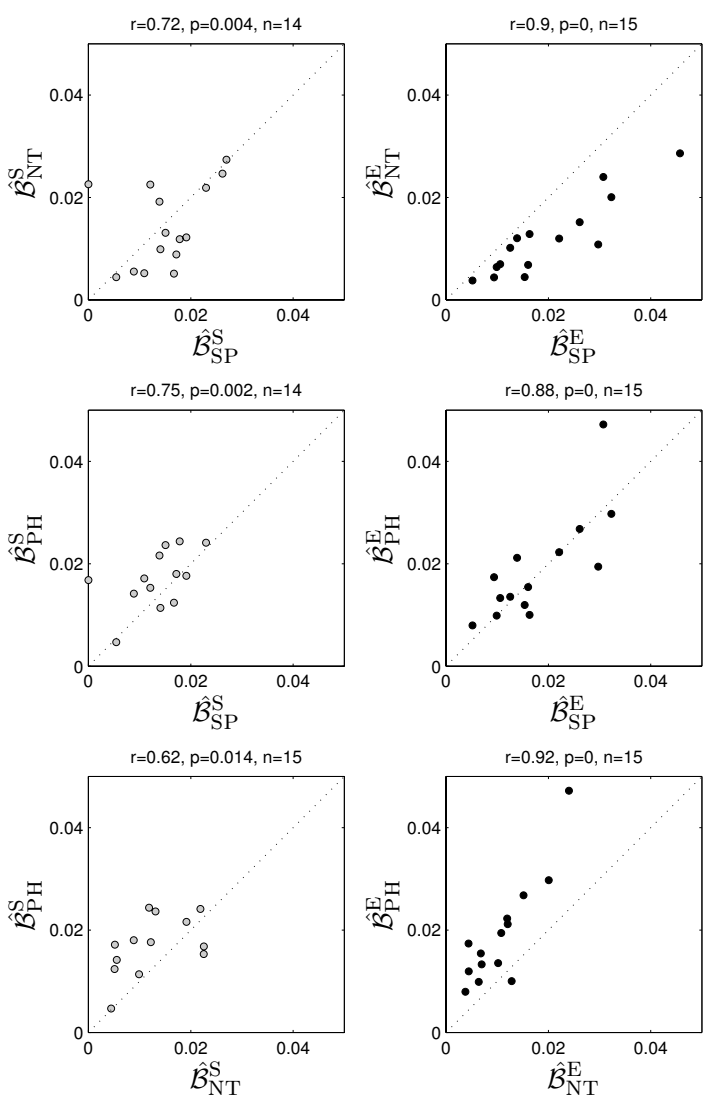

Figure 4. Dispersion diagrams comparing $\hat{\mathcal{B}}^{\text {s }}$ (grey) and $\hat{\mathcal{B}}^{\mathrm{E}}$ (black) in SP, NT and PH conditions.

The value of $\delta$ is a trade-off between $N$ and the $\hat{\boldsymbol{\epsilon}}$ standard deviation (inversely related with $r$ ), with higher $N$ (or $r$ ) leading to lower $\delta$ (i.e, higher precision). As illustrated in Fig. 5, the less restrictive thresholds for BE identification lead to $r_{\mathrm{SP}}^{\mathrm{E}}<r_{\mathrm{SP}}^{\mathrm{S}}$ and $N_{\mathrm{SP}}^{\mathrm{E}}>N_{\mathrm{SP}}^{\mathrm{S}}$ so that $\delta_{\mathrm{SP}}^{\mathrm{E}}<\delta_{\mathrm{SP}}^{\mathrm{S}}$ in $8 / 12$ cases and the mean paired differences $\delta_{\mathrm{SP}}^{\mathrm{E}}-\delta_{\mathrm{SP}}^{\mathrm{S}}$ do not differ from zero $(\mathrm{p}>0.1)$. The $\delta_{\mathrm{SP}}$ value decays with increasing $N_{\mathrm{SP}}$ and $r_{\mathrm{SP}}$ remaining close to 0.8 . Therefore, $\delta_{\mathrm{SP}}$ is more influenced by $N_{\mathrm{SP}}$ than by $r_{\mathrm{SP}}$. For NT and PH, $N$ is lower and $r$ is higher than in SP. For BSs, $\delta_{\mathrm{NT}}^{\mathrm{s}}$ and $\delta_{\mathrm{PH}}^{\mathrm{s}}$ have the same range as $\delta_{\mathrm{SP}}^{\mathrm{S}}$ and $\delta_{\mathrm{SP}}^{\mathrm{S}}<\delta_{\mathrm{NT}}^{\mathrm{s}}$ in $5 / 10$ of the cases and $\delta_{\mathrm{SP}}^{\mathrm{S}}<\delta_{\mathrm{PH}}^{\mathrm{s}}$ in $5 / 10$. For BEs, the slightly higher $r^{\mathrm{E}}$ and much lower $N^{\mathrm{E}}$ in NT and $\mathrm{PH}$ in comparison to SP lead to $\delta_{\mathrm{SP}}^{\mathrm{E}}<\delta_{\mathrm{NT}}^{\mathrm{E}}$ in $13 / 15$ cases and $\delta_{\mathrm{SP}}^{\mathrm{E}}<\delta_{\mathrm{PH}}^{\mathrm{E}}$ in $12 / 15$. That is, no precision improvement is achieved with the druginduced protocol: for BSs, the lower $N^{\mathrm{S}}$ is balanced by the increased $r^{\mathrm{s}}$, so that the $\delta^{\mathrm{s}}$ is maintained; for BEs, the 

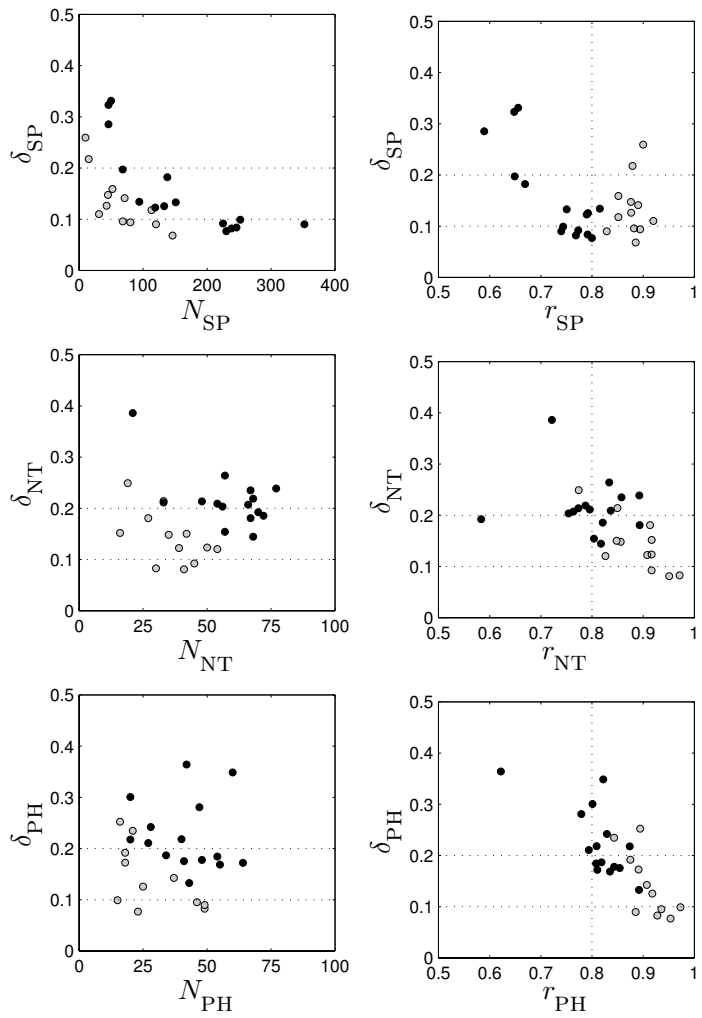

Figure 5. Plot of $\delta$ as a function of $N$ and $r$ for $\hat{\mathcal{B}}^{\mathrm{s}}$ (grey) and $\hat{\mathcal{B}}^{\mathrm{E}}$ (black) in SP, NT and PH conditions.

higher $r^{\mathrm{E}}$ does not pay off the much lower $N^{\mathrm{E}}$, so that $\delta^{\mathrm{E}}$ is higher in either NT and PH when compared to SP.

In SP there is no time span constrain as in drug-induced experiments (especially if using a bolus instead of a continuous infusion). Therefore, the possibility of increase $N$ in SP is an advantage over increase $r$ in a drug-induced protocol: in SP, higher $N$ requires longer recordings whereas higher $r$ requires higher $x_{\mathrm{SBP}}-x_{\mathrm{RR}}$ correlation (only obtained with stimulation). In SP, $r_{\mathrm{SP}}^{\mathrm{S}}$ is higher than 0.8 and $r_{\mathrm{SP}}^{\mathrm{E}}$ is constrained to be lower than 0.8 (with $r_{\min }=0.8$ for BE identification, $r^{\mathrm{E}}$ will tend to be 0.8 in SP stationary recordings [6]). In contrast, the longer the recordings the higher the $N^{\mathrm{E}}$ and $N^{\mathrm{S}}$ difference is. Therefore, if the SP stationary conditions are satisfied (i.e, $r_{\mathrm{sp}}^{\mathrm{E}}$ close to 0.8 ), it is expected that $\hat{\mathcal{B}}_{\mathrm{SP}}^{\mathrm{E}}$ outperforms $\hat{\mathcal{B}}_{\mathrm{SP}}^{\mathrm{S}}$ in precision.

\section{Conclusions}

In this work, BRS analysis from BSs and BEs is further compared using spontaneous and drug induced data. Besides its non invasive nature, the spontaneous BRS estimates are preferred to drug induced estimates, as no precision improvements are achieved with the modified Oxford method. The BRS analysis from BEs distinguishes better the different conditions, including for BRS estimates with lower precision. Also, with the use of BEs instead of BSs in spontaneous condition, an improvement of the BRS estimate precision is achieved, for the same recording length.

\section{Acknowledgements}

S Gouveia acknowledges the grant SFRH/BD/18894/2004 by FCT/ESF. This work was partially supported by CMUP (financed by FCT Portugal through POCI2010/POCTI/POSI programmes, with national and CSF funds) and TEC200768076-C02-02 from CICYT/FEDER Spain.

\section{References}

[1] Sleight P. New methods for risk stratification in patients after myocardial infarction: Autonomic control and substrate sensitivity. J Am Coll Cardiol 2007;50:2291-2293.

[2] La Rovere M, Pinna G, Raczak G. Baroreflex sensitivity: Measurement and clinical implications. Ann Noninvasive Electrocardiol 2008;13(2):191-207.

[3] Parlow J, Viale J, Annat G, Hughson R, Quintin L. Spontaneous cardiac baroreflex in humans: comparison with druginduced responses. Hypertension 1995;25:1058-1068.

[4] Mortara A, La Rovere M, Pinna G, Parziale P, Maestri R, Capomolla S, Opasich C, Cobelli F, Tavazzi L. Depressed arterial baroreflex sensitivity and not reduced heart rate variability identifies patients with chronic heart failure and nonsustained ventricular tachycardia. Am Heart J Nov 1997;134(5 Pt 1):879-888.

[5] Bertineri G, Rienzo MD, Cavallazzi A. Evaluation of baroreceptor reflex by blood pressure monitoring in unanesthetized cats. Am J Physiol 1988;254:H377-H383.

[6] Gouveia S, Rocha AP, Laguna P, Lago P. Improved time domain BRS assessment with the use of baroreflex events. Proc Comput Cardiol 2007;34:813-816.

[7] Wilcox R. Introduction to robust estimation and hypothesis testing. 2nd edition. Elsevier Academic Press, 2005.

[8] Shumway R, Stoffer D. Time series analysis and its applications. 2nd edition. Springer, 1993.

[9] Gujic M, Laude D, Houssière A, Beloka S, Argacha J, Adamopoulos D, Xhaët O, Elghozi J, van de Borne P. Differential aspects of metaboreceptor and chemoreceptor activation on sympathetic and cardiac baroreflex control following exercise in hypoxia in human. J Physiol 2007; 585(1):165-174.

[10] Brunton L, Blumenthal D, Buxton I. Goodman and Gilman Manual of Pharmacology and Therapeutics. McGraw-Hill, 2007.

[11] Pickering T, Gribbin B, Sleight P. Comparison of the reflex heart rate response to rising and falling arterial pressure in man. Cardiovasc Res 1972;6(3):277-283.

Address for correspondence:

Sónia Gouveia

Dep Matemática Aplicada, Faculdade Ciências Univ Porto Rua do Campo Alegre, 687; 4169-007, Porto, Portugal.

E-mail address: sagouvei@fc.up.pt. 